NOTE ICHTYOLOGIQUE

\title{
SUR LA PRÉSENCE EN FRANCE DE PSEUDORASBORA PARVA (SCHLEGEL, 1842)
}

\author{
J. ALLARDI (1) et F. CHANCEREL (2)
}

(1) CEMAGREF, Division Qualité des Eaux Pêche et Pisciculture, 14, avenue de Saint-Mandé, 75012 PARIS.

(2) Délégation Régionale du C.S.P., 112, Faubourg de la Cueille, 86000 POITIERS.

Reçu le 14 décembre 1987

Accepté le 6 janvier 1988

Received 14 December, 1987 Accepted 6 January, 1988

\section{RÉSUMÉ}

Les auteurs décrivent une espèce nouvelle pour la faune de France: Pseudorasbora parva (SCHLEGEL, 1842). Ils donnent des informations sur son aire de répartition actuelle et sur son origine en France.

\section{ON THE PRESENCE IN FRANCE OF PSEUDORASBORA PARVA \\ (SCHLEGEL, 1842)}

\section{SUMMARY}

The authors describe a new record for the french ichtyofaune: Pseudorasbora parva (SCHLEGEL, 1842). They give informations about the present distribution and origin in France.

C'est en 1983 que la Délégation Régionale du CONSEIL SUPÉRIEUR DE LA PÊCHE de POITIERS nous faisait parvenir un poisson ne figurant pas dans l'ouvrage de référence de SPILLMANN (1961). Cet unique spécimen avait été capturé dans la Vègre (affluent de la Sarthe) sur la commune de Tennie.

\section{DESCRIPTION DE L'ESPÈCE :}

II s'agit d'un petit poisson de la famille des Cyprinidés à nageoires anale et dorsale courtes. La bouche dépourvue de barbillons présente un prognathisme inférieur notable. La fente buccale est orientée vers le haut, et presque verticale lorsque la bouche est fermée (figure 1).

Le faible nombre d'écailles $(\approx 35)$ le long de la ligne latérale permet de distinguer le genre Pseudorasbora (BLEECKER, 1860) auquel appartient ce poisson des genres Leuciscus, Telestes et Rutilus.

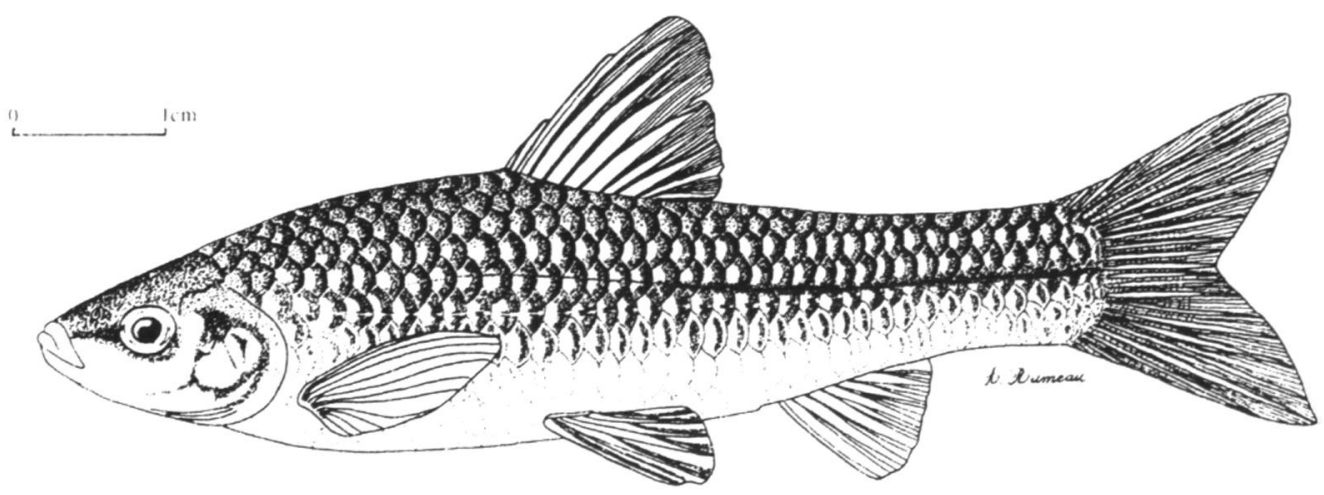

Figure 1 : Pseudorasbora parva (Schlegel, 1842). 
Les écailles relativement grandes ont le bord postérieur souligné par un grand nombre de mélanophores rappelant un peu la robe du chevaine. Lorsque les poissons sont vivants, on distingue sur le flanc des reflets jaune d'or ainsi qu'une bande latérale foncée seule visible après la mort.

Dans le tableau I sont données les principales mesures effectuées sur le premier spécimen de la Vègre ainsi que sur un spécimen en provenance d'un étang dans le département du Doubs.

Ces mesures sont comparées à celles données dans l'ouvrage de BANARESCU (1964) que nous avons utilisé pour déterminer nos individus.

II existe une incertitude en ce qui concerne le nom exact de l'espèce ; BANARESCU (1964) donne Pseudorasbora parva (SCHLEGEL, 1842), mais la plupart des auteurs donnent Leuciscus parvus (TEMMINCK et SCHLEGEL, 1846) (DAGET, com. pers.). La description originale de l'espèce se trouve dans l'ouvrage d'OKADA (1959-1960).

\begin{tabular}{|c|c|c|c|c|c|c|}
\hline & & $*$ LS & & x LS & & 3 LS \\
\hline ט & & & & & & \\
\hline Longueur totale & 58.7 & 120.5 & 75 & 125 & 117 & $-121,3$ \\
\hline Longueur standard (LS) & 48,7 & 100 & 60 & 100 & & - $\quad$ \\
\hline Hauteur max. du corps & 13,5 & 27.7 & 14 & 23 & & - \\
\hline Hauteur min. du corps & 6 & 12,3 & 7 & 12 & & - \\
\hline Longueur pédoncule caudal & 11.5 & 23,6 & 13 & 22 & 20 & -26 \\
\hline Longueur de la tête & 12,7 & 26,0 & 15 & 25 & 22,8 & -27.5 \\
\hline Longueur du museau & 4.7 & 9,6 & 4,8 & 8 & & - $\quad$ \\
\hline Diamètre de loeil & 3 & 6,1 & 3 & 5 & & - \\
\hline Diamètre de l'oejl/longueur de la téte & 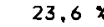 & & $20 \%$ & & & \\
\hline Distance prédorsale & 24,5 & 50.2 & 27 & 47 & 45 & -50.3 \\
\hline Distance préanale & 34 & 69,8 & 41,5 & 69 & & - \\
\hline Longueur dorsale & 6.5 & 13,3 & 12,5 & 21 & & - \\
\hline Ecailles ligne latérale (nombre) & $\approx 35$ & & 35 & & 34 & -38 \\
\hline Rayons dorsale (nombre) & III $/ 7$ & & III $/ 7$ & & I I & \\
\hline
\end{tabular}

Tableau I : Liste des proportions corporelles et données méristiques

1 - Exemplaire provenant de la Vègre.

2 - Exemplaire provenant d'un étang dans le Doubs.

3 - Valeurs données par BANARESCU (1964).

Table 1 : Measurements and meristic counts

1 - Sample from the Vère River

2 - Sample from a fishpond in the Doubs

3 - Values from Banarescu (1964)

\section{ORIGINE DE L'ESPĖCE :}

Cette espèce originaire de l'Asie du Sud-Est (Japon) est signalèe en Chine; elle a été introduite en Roumanie en 1960, sous la forme d'alevins mélangés à d'autres cyprinidés en provenance du bassin du fleuve Yang Tseu Kiang (BANARESCU, 1964). Dans ce pays, elle se reproduit dès le printemps 1961 dans des étangs, d'où elle a pu gagner des rivières voisines. Considérée comme indésirable, cette espèce est maintenant largement répandue dans le sud de la Roumanie (BACALBASA DROBOVICI, 1984).

Nous ne connaissons pas avec précision les conditions d'introduction de cette espèce en France. D'après le Garde-Pêche commissionné LEROUX: "ce poisson aurait été introduit en 1978-1979 dans la région de Beaumont-sur-Sarthe (département de la Sarthe). Les premiers sujets ont été vus en juin 1980 (individus de 7 à $9 \mathrm{~cm}$ ) à l'aval du barrage de Vivoin. Ces poissons pêchés par des enfants mordaient à tous les appâts".

Actuellement, l'espèce est signalée dans la Vègre, dans plusieurs étangs du Doubs, dans l'Yzeron (affluent de la rive droite du Rhône), dans le département du Rhône (SOUCHON, com. pers.), puis récemment dans des étangs du Haut-Rhin à proximité de Hagenbach.

\section{DISCUSSION :}

L'introduction volontaire ou involontaire d'une espèce nouvelle constitue toujours un risque dont nous ne pouvons pas prévoir les conséquences (risque de compétition alimentaire avec d'autres espèces plus intéressantes, dont la carpe dans le cas présent, risque d'introduction d'agents pathogènes par exemple). Actuellement, les organismes nationaux et internationaux tendent à réglementer au maximum le transfert d'espèces de pays à pays. 
Bull. Fr. Pêche Piscic. (1988) 308: $35-37 \quad-37-$

Cependant, l'importance actuelle des échanges rend difficile, si ce n'est impossible, un contrôle systematique des introductions.

La capture, depuis plusieurs années, de Pseudorasbora parva dans les cours d'eau (Yzeron, Vègre) ou des étangs (Doubs, Haut-Rhin) prouve que cette espèce est actuellement acclimatée dans notre pays et doit être ajoutée à la liste déjà longue des espèces introduites en France (ALLARDI, 1984).

\section{REMERCIEMENTS :}

Les auteurs remercient Monsieur le Professeur J. DAGET du Muséum National d'Histoire Naturelle de PARIS qui a confirmé notre détermination, ainsi que Monsieur SOUCHON et les Gardes-Pêche commissionnés de l'Administration BOHN et GAMBERI, pour leurs informations. Le dessin de Pseudorasbora parva a été réalisé par le Garde-Chef A. RUMEAU.

\section{RÉFÉRENCES :}

ALLARDI J., 1984. Introduction et acclimatation des poissons d'eau douce en France. Historique et bilan. EIFAC Tech. Paper 42-2. 427-435.

BACALBASA DOBROVICI N., 1984. Introduction de nouvelles espèces de poissons dans les pêcheries d'eau douce de la Roumanie. EIFAC Tech. Paper 42-2. 458-465.

BANARESCU P., 1964. Fauna Republicii Populare. Pisces Osteichthys XIII. 559 p.

OKADA Y., 1959-1960. Studies on the freshwater fisches of Japan. Tsu Mie Prefec. Ed. $860 \mathrm{p}$. SPILLMANN C.J., 1961. Poissons d'eau douce. Faune de France 65. P. Lechevalier Ed. 303 p. 